\title{
Differences in the Detection of Cryptosporidium and Isospora (Cystoisospora) Oocysts According to the Fecal Concentration or Staining Method Used in a Clinical Laboratory
}

\author{
Author(s): Flávia T. F. Pacheco, Renata K. N. R. Silva, Adson S. Martins, Ricardo R. Oliveira, Neuza M. \\ Alcântara-Neves, Moacir P. Silva, Neci M. Soares, and Márcia C. A. Teixeira \\ Source: Journal of Parasitology, 99(6):1002-1008. \\ Published By: American Society of Parasitologists \\ DOI: http://dx.doi.org/10.1645/12-33.1 \\ URL: http://www.bioone.org/doi/full/10.1645/12-33.1
}

BioOne (www.bioone.org) is a nonprofit, online aggregation of core research in the biological, ecological, and environmental sciences. BioOne provides a sustainable online platform for over 170 journals and books published by nonprofit societies, associations, museums, institutions, and presses.

Your use of this PDF, the BioOne Web site, and all posted and associated content indicates your acceptance of BioOne's Terms of Use, available at www.bioone.org/page/terms_of_use.

Usage of BioOne content is strictly limited to personal, educational, and non-commercial use. Commercial inquiries or rights and permissions requests should be directed to the individual publisher as copyright holder. 


\title{
DIFFERENCES IN THE DETECTION OF CRYPTOSPORIDIUM AND ISOSPORA (CYSTOISOSPORA) OOCYSTS ACCORDING TO THE FECAL CONCENTRATION OR STAINING METHOD USED IN A CLINICAL LABORATORY
}

\author{
Flávia T. F. Pacheco, Renata K. N. R. Silva, Adson S. Martins, Ricardo R. Oliveira, Neuza M. Alcântara-Neves*, \\ Moacir P. Silva*†, Neci M. Soares, and Márcia C. A. Teixeira \\ Faculdade de Farmácia, Universidade Federal da Bahia, Salvador, Bahia, Brazil, 40170-115. Correspondence should be sent to: marciat@ufba.br
}

\begin{abstract}
Despite the availability of many parasitological methods for detection of Cryptosporidium and Isospora (Cystoisospora) belli in fecal samples, there are uncertainties about the accuracy of these techniques in laboratory practice. In this study, 27 formalinfixed positive stool samples for Cryptosporidium and 15 for I. belli were analyzed by 2 concentration methods, sedimentation by centrifugation (SC) and formalin-ethyl acetate (FE), and by 3 tintorial techniques, modified Ziehl-Neelsen (ZN), safranin (SF), and auramine (AR). No significant differences were observed on Cryptosporidium identification between concentration methods, while a significantly higher number of $I$. belli oocysts $(P<0.0001)$ was detected in fecal smears concentrated by the SC than by the FE method. Fecal samples processed by FE produced a median oocyst loss to the fatty ring of $34.8 \%$ for Cryptosporidium and $45.4 \%$ for I. belli. However, FE concentration provided $63 \%$ of Cryptosporidium and $100 \%$ of $I$. belli slides classified as superior for microscopic examination. Regarding the efficiency of staining methods, a more significant detection of Cryptosporidium oocysts was observed in fecal smears stained by ZN $(P<0.01)$ or AR $(P<0.05)$ than by the SF method. Regular to high-quality slides for microscopic examination were mostly observed in fecal smears stained with AR or ZN for Cryptosporidium and with SF or ZN for $I$. belli. This study suggests a great variability in oocyst power detection by routine parasitological methods, and that the most frequent intestinal coccidians in humans have specific requirements for concentration and staining.
\end{abstract}

Cryptosporidium spp. and Isospora (Cystoisospora) belli are the most common opportunistic enteroparasites in patients with Acquired Immunodeficiency Deficiency Syndrome (AIDS) and are considered important etiological agents of chronic diarrhea in these subjects (Vignesh et al., 2007; Gupta et al., 2008; Hanscheid et al., 2008; Kulkarni et al., 2009; Alemu et al., 2011; Cardoso et al., 2011). Both chronic coccidial infections in AIDS patients may progress to a debilitating status and even to death (Lindsay et al., 1997; Cimerman et al., 1999; Chalmers and Davies, 2010). In developing countries, Cryptosporidium infection is also widespread in children under 5-yr-old and may cause acute or persistent diarrhea when associated with malnutrition (Huang et al., 2004; Agnamey et al., 2010; Moyo et al., 2011).

There are several immunological methods for the detection of Cryptosporidium in fecal samples such as enzyme immunoassays (ELISA) (Garcia and Shimizu, 1997; Weitzel et al., 2006; Jayalakshmi et al., 2008; Elgun and Koltas, 2011), direct immunofluorescence, and rapid immunological tests (Magi et al., 2006; Collinet-Adler and Ward, 2010; Teixeira et al., 2011). Molecular biology techniques have been used for both Cryptosporidium and I. belli identification such as the polymerase chain reaction (PCR) and its variations, which are mainly applied in research (Magi et al., 2006; ten Hove et al., 2008; Skotarczak, 2010) due to the high costs incurred by the routine laboratory. Therefore, the most widely used methods are those that enable visualization of oocysts in feces without determining the species involved and using staining techniques of fecal smears on glass slides based on the acid-resistance property of coccidian oocysts (Lindsay et al., 1997; Magi et al., 2006; Harrington, 2008).

Before staining fecal smears, concentration methods are strongly recommended, especially for the diagnosis of coccidiosis

Received 1 August 2012; revised 25 April 2013; accepted 19 June 2013. * Instituto de Ciências da Saúde, Universidade Federal da Bahia, Bahia, Brazil 40110-902.

† Laboratório Central de Saúde Pública Professor Gonçalo Moniz, Salvador, Bahia, Brazil 40295-001.

DOI: 10.1645/12-33.1 in asymptomatic patients. The techniques can be based on flotation or sedimentation processes such as sucrose flotation methods or formalin-ether sedimentation, respectively (Weber et al., 1991; Clavel et al., 1996; Kar et al., 2011). After stool concentration, fecal smears can be stained by a wide variety of techniques, although the variants of Ziehl-Neelsen (Henriksen and Pohlenz, 1981; Huber et al., 2004), safranin (Baxby et al., 1984; Huber et al., 2004) and auramine (Lindsay et al., 1997; Hanscheid et al., 2008) are the most frequently used either in routine or research laboratories.

Despite the diversity of existing parasitological methods for detection of Cryptosporidium and I. belli in stools, there are uncertainties about the selection and standardization of diagnostic protocols for implementation in clinical laboratories. Therefore, this study aimed to compare parasitological methods regularly used in clinical laboratories for concentration and staining of Cryptosporidium and I. belli in order to identify the most efficient tool to detect both parasites in fecal samples.

\section{MATERIALS AND METHODS}

\section{Fecal samples}

To compare the efficacy of oocyst detection by different concentration and staining techniques, 27 Cryptosporidium and 15 I. belli formalin-fixed stool samples, from naturally occurring infections, were individually tested. The Cryptosporidium and I. belli positive samples used in this study were obtained from the Central Laboratory of Public Health in the state of Bahia (Brazil) and diagnosed during the routine analysis of fecal samples from AIDS patients or from children with diarrhea in the Parasitology Laboratory. Briefly, at the time of diagnosis, 2 grams of feces were homogenized in $50 \mathrm{ml}$ of water and filtered through gauze. Fecal suspension was then centrifuged in a $15-\mathrm{ml}$ tube for $2 \mathrm{~min}$ at $400 \mathrm{~g}$. The supernatant was discarded and the fecal pellet was re-suspended in $10 \%$ formalin. The centrifugation process was repeated and the sediment was examined by preparing wet mounts and by staining fecal smears. Isospora samples were identified in iodine wet mount, by staining smears with Ziehl-Neelsen and safranin, or both. Cryptosporidium samples were diagnosed by an ELISA commercial kit (Wampole Cryptosporidium II, TECHLAB, Blacksburg, Virginia) for coproantigens detection using fresh or frozen fecal aliquots; thereafter, fecal smears were stained with ZiehlNeelsen and safranin to confirm the presence of oocysts. For our study, the only information obtained was if the sample had microscopically visible oocysts, regardless of the number of parasites or method used for 
diagnosis. There was no access to any further information about patients. This study was approved by the Committee of Ethics in Research of the Department of Health of Bahia.

\section{Quantitative evaluation of parasitological methods for concentration and staining of Cryptosporidium and Isospora belli oocysts}

Positive fecal samples, previously sieved and fixed in formalin, were initially analyzed by 2 oocyst concentration techniques: sedimentation by centrifugation (SC) and formalin-ethyl acetate (FE) (Truant et al., 1981). Three fecal smears were made for each sample, 1 from the SC-sediment and 2 from FE (a smear from the FE-ring with fatty debris and the other from the FE-sediment), and then stained with a modified Ziehl-Neelsen technique (Henriksen and Pohlenz, 1981). Thereafter, to evaluate oocyst coloration methods, positive samples concentrated by SC were stained by 3 techniques: modified Ziehl-Neelsen (ZN) (Henriksen and Pohlenz, 1981), safranin (SF) (Baxby et al., 1984), and auramine (AR) (Winn et al., 2006). To make the methods comparable, we used a standard volume of $5 \mathrm{ml}$ of formalin-fixed fecal samples, centrifuged tubes for $2 \mathrm{~min}$ at $400 \mathrm{~g}$, and prepared smears using $25 \mu \mathrm{l}$ of concentrated sample spread over a glass slide in an area of approximately $2.5 \times 1.5 \mathrm{~cm}$. Fecal smears were prepared in the lab by a group different from the slide readers. Samples were identified with new numbers and randomly distributed among microscopists in an attempt to avoid the reading of matched samples and, thus, hamper comparisons; e.g., microscopist A received the slide of Cryptosporidium-safranin but not the Cryptosporidium-Ziehl. For comparative analysis among concentration and staining procedures for Cryptosporidium and I. belli, 20 microscopic fields of fecal smears were examined independently by 2 microscopists with a magnification of $\times 400$. In case there was variance greater than $10 \%$ in the number of oocysts counted, or a lack of agreement in qualitative classification of methods tested, a third observer also checked the slides. The efficiency of the methods was assessed quantitatively by the total number of oocysts detected in 20 microscopic fields of fecal smears. Proportions of samples with higher number of oocysts, according to the concentration or staining method used, are presented as percentages. It is worth mentioning that for diagnostic purposes, considering the heterogeneity in oocysts excretion by infected patients, the entire fecal smear should be examined. In addition, microscopists should not rule out the possibility of coccidian (Cryptosporidium, Isospora, and Cyclospora) mixed infections. Therefore, even if a specific coccidian is found in large amounts, it is still advisable to read the entire smear. In our study we worked with a panel of previously identified positive samples with easily detectable oocysts so that the count of 20 fields was sufficient for comparison studies. Moreover, we evaluated the potential loss of Cryptosporidium and I. belli oocysts in the ring of fat debris, a layer normally produced in the FE concentration technique, and determined if this loss was significant enough to influence the performance of the method.

\section{Qualitative evaluation of parasitological methods for concentration and staining Cryptosporidium and Isospora belli oocysts}

Qualitative assessment of parasitological methods was achieved by evaluating the intensity of dye uptake by oocysts, its distinction from the background, and the presence of stained nonrelevant residues from stool. In this study, the superior diagnostic method was defined as the one capable of easily discriminating the oocysts from surrounding areas in fecal smears and incapable of dying other fecal elements such as yeast cells, pollen grains, or digested food residues. Moreover, the ideal method for coccidian diagnosis also should not produce precipitation of dye particles, which is a main concern for Cryptosporidium diagnosis, especially when sizes and shapes of residues are similar to the oocysts Considering the evaluation of 20 microscopic fields to analyze these nonquantitative variables, stained fecal smears with more than 10 fields $(>50 \%)$ with any "undesirable" characteristics (as described above) were classified as inferior, those between 5-10 fields $(25-50 \%)$ as regular, and the remaining, with less than 5 fields $(<25 \%)$, as superior. Results of qualitative evaluation were presented as the percentage of samples fitted in each method classification.

\section{Statistical analysis}

Statistical analysis was performed using GraphPad Prism 5.0 software (GraphPad Software, San Diego, California). The d'Agostino-Pearson
TABLE I. Median (range) of oocysts detected in 20 microscopic fields of Cryptosporidium- and Isospora belli-positive fecal smears, concentrated by sedimentation-centrifugation (SC) or formalin-ethyl acetate (FE) methods.

\begin{tabular}{lcccc}
\hline & & \multicolumn{2}{c}{ Concentration method* } & \\
\cline { 3 - 4 } \multicolumn{1}{c}{ Coccidia } & $\mathrm{n}$ & $\mathrm{SC}$ & $\mathrm{FE}$ & $P$ \\
\hline Cryptosporidium $\mathrm{sp}$. & 27 & $380(16-4,038)$ & $230(6-4,554)$ & 0.714 \\
Isospora belli & 15 & $68(5-318)$ & $28(1-137)$ & $<0.0001^{*}$ \\
\hline
\end{tabular}

* Significant difference in the numbers of Isospora belli oocysts detected by SC compared to the FE concentration method (Wilcoxon nonparametric test).

omnibus normality test was used to designate the most appropriate statistical test for each evaluation. Because the number of oocysts detected in 20 microscopic fields did not follow the normal distribution, this variable was expressed as median, maximum, and minimum observed values. A total of 27 and 15 stool samples positive for Cryptosporidium and Isospora belli, respectively, were included. Each sample was tested by 2 fecal concentrations and 3 different staining methods. The nonparametric Wilcoxon matched pairs test was used to compare number of Cryptosporidium or Isospora oocysts detected in the same samples by SC and FE methods. The nonparametric Friedman's 2-way ANOVA test was used to compare the number of Cryptosporidium or Isospora oocysts obtained by 3 different staining methods in order to take into account the matched design of the study. The chi-square test was used to compare proportions among staining or fecal concentration methods. The significant alpha level adopted was 0.05 .

\section{RESULTS}

The number of parasites in preserved stools had a great variability, as shown by the large range of Cryptosporidium oocysts identified by SC or FE. A significantly higher number of $I$. belli oocysts were detected in fecal smears concentrated by the SC when compared to the FE method $(P<0.0001$; Table I). Analyzing the samples individually, the SC identified more Cryptosporidium and Isospora oocysts in 16 of $27(59.3 \%)$ and in 15 of $15(100 \% ; P<0.01)$ samples, respectively, in contrast to the FE method, which failed in concentrating Isospora oocysts (Fig. 1A).

The evaluation of nonquantitative microscopic parameters classified fecal smears concentrated by FE mostly as superior (n $=17 ; 63 \%$ ) for diagnosis of Cryptosporidium as well as for I. belli (n $=12 ; 80 \% ; P>0.05)$ (Fig. 1B). The fecal concentration by SC method showed a higher variability in qualitative analysis for Cryptosporidium than for I. belli diagnosis, with $25.9 \%$ and $12.5 \%$ of smears classified as inferior, respectively $(P>0.05)$ (Fig. $1 \mathrm{~B})$.

The assessment of oocyst loss to the fecal fatty plug produced by the FE concentration technique showed the presence of parasites in fatty layer smears in $96.3 \%(26 / 27)$ of Cryptosporidium and in $93.3 \%(14 / 15)$ of $I$. belli samples. Moreover, the number of oocysts was even higher in this area than in the sediment for $25.9 \%(7 / 27)$ and $46.6 \%(7 / 15)$ of the samples analyzed, respectively. Considering the total count, the median oocyst loss to the fatty ring was $34.8 \%$ (ranging from 0 to $96.7 \%$ ) for Cryptosporidium and $45.4 \%$ (ranging from 0 to $84.4 \%$ ) for $I$. belli. A significantly higher distribution of Cryptosporidium oocysts in the sediment rather than in the fatty debris area $(P=$ 0.0042 ) was observed. This result differed from I. belli samples, 

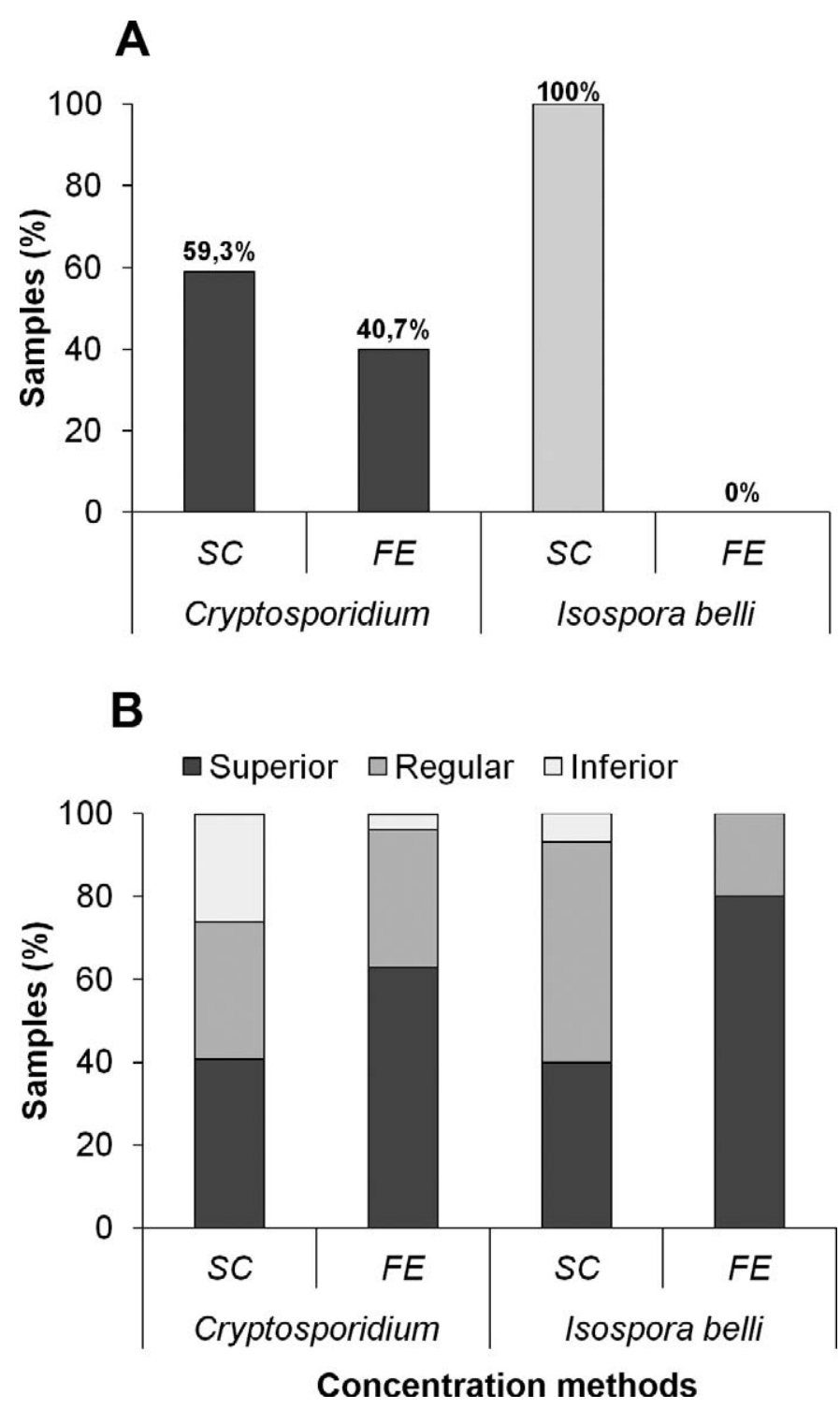

FIgURE 1. (A) Quantitative evaluation of fecal concentration techniques.Twenty-seven Cryptosporidium and 15 Isospora belli fecal samples were concentrated by the SC or FE techniques and stained by modified Ziehl-Neelsen. Columns represent the percentual of samples showing a higher number of oocysts, analyzed by examining 20 microscopic fields. (B) Qualitative classification of concentration methods. Proportion of fecal smears classified as superior, regular, or inferior according to the microscopic parameters described in Materials and Methods.

which displayed a similar distribution of oocysts in both areas $(P$ $=0.35)$ (Table II).

The performance of dying techniques varied according to the parasite tested. A significantly higher detection of Cryptosporidium oocysts was observed in fecal smears stained by $\mathrm{ZN}(P<$ $0.01)$ or AR $(P<0.05)$ than those stained with the SF method (Table III). Samples positive for I. belli showed no statistical differences in the number of oocysts detected among the staining methods, although a higher median of oocysts was observed in $\mathrm{ZN}$-fecal smears (Table III). Moreover, more than half of Cryptosporidium $(57.7 \%)$ and I. belli $(53.7 \%)$ slides showed greater number of oocysts when stained with $\mathrm{ZN}$ (Fig. 2A). In
TABLE II. Median and range of oocysts of Cryptosporidium and Isospora belli detected in 20 microscopic fields of fecal smears according to the area of FE-concentration method examined.

\begin{tabular}{lllcl}
\hline & & \multicolumn{2}{c}{ Area of fecal debris examined } & \\
\cline { 3 - 4 } \multicolumn{1}{c}{ Coccidia } & $\mathrm{n}$ & \multicolumn{1}{c}{ FE-FR } & FE-SE & \multicolumn{1}{c}{$P$} \\
\hline Cryptosporidium $\mathrm{sp}$. & 27 & $81(0-1,502)$ & $230(6-4,554)$ & $0.0042^{*}$ \\
Isospora belli & 15 & $31(0-189)$ & $28(1-137)$ & 0.35 \\
\hline
\end{tabular}

* Significant difference in the numbers of Cryptosporidium oocysts in the sediment (FE-SE) compared to the fatty ring area (FE-FR) of fecal smears after FE concentration (Wilcoxon non-parametric test).

contrast, few samples had more oocysts of Cryptosporidium $(3.8 \%)$ or I. belli $(13.0 \%)$ detected by SF (Fig. 2 A).

The qualitative assessment of microscopic characteristics of stained fecal smears fitted the majority of Cryptosporidium $(\mathrm{n}=$ $21 ; 80.8 \%)$ or I. belli $(\mathrm{n}=10 ; 66.7 \% ; P>0.05) \mathrm{ZN}$-slides in the superior classification (Fig. 2B). All AR-stained smears displayed a high quality for Cryptosporidium identification. The slides stained using this technique had little or no impurity, clearly showing bright fluorescent oocysts, in contrast to SF technique which frequently presented fecal debris nonspecifically stained or dye residues with size and shapes similar to Cryptosporidium oocysts. Nonetheless, only 3 AR slides were classified as superior for $I$. belli identification $(P<0.0001)$. The SF staining produced a higher proportion of slides classified as inferior for both coccidians (Fig. 2B; $P<0.05$ ). Figure 3 presents the microscopic characteristics of stained fecal smears used for qualitative classification of methods.

\section{DISCUSSION}

Because of the irregular daily excretion and heterogeneous distribution of oocysts in stool samples, it is highly recommended that parasites are concentrated by centrifugation or fluctuation methods before staining fecal smears. Concentration methods aim to eliminate confusing fecal debris and increase the chances of finding protozoa oocysts, especially in asymptomatic individuals with low parasite discharge (Casemore, 1991; Huber et al., 2003, 2004). The formalin-ether or formalin-ethyl acetate techniques have the advantage of removing fats and fibers present in stools and are highly recommended for the diagnosis of human coccidians. However, comparative studies using this technique have shown inconsistent results (Casemore et al., 1985; McNabb et al., 1985; Clavel et al., 1996; Kuczynska and Shelton, 1999; Huber et al., 2003).

In our study, positive stool homogenates processed by SC produced higher counts of oocysts in microscopic fields than did the concentration using FE; this was particularly significant for $I$. belli detection, with all samples showing more oocysts in smears concentrated by the former. On the other hand, stools processed by FE had smaller amounts of impurities, as expected, highlighting the presence of oocysts in the smear and, therefore, fitting in the superior or regular qualitative classification. To assess the potential loss of oocysts during FE concentration, we simultaneously examined the fat plug and the sediment area. Distribution of Cryptosporidium or I. belli oocysts in the fat ring varied considerably, ranging from 0 to $96.7 \%$ or 0 to $84.4 \%$, respectively, of the total number of identified oocysts depending 
TABle III. Median and range of oocysts of Cryptosporidium and Isospora belli detected in 20 microscopic fields of fecal smears according to the stain method used.

\begin{tabular}{|c|c|c|c|c|c|c|c|}
\hline \multirow[b]{2}{*}{ Coccidia } & \multirow[b]{2}{*}{$\mathrm{n}$} & \multicolumn{3}{|c|}{ Stain method } & \multicolumn{3}{|c|}{$P$} \\
\hline & & $\mathrm{ZN}$ & $\mathrm{SF}$ & AR & $\mathrm{ZN} \times \mathrm{SF}$ & $\mathrm{ZN} \times \mathrm{AR}$ & $\mathrm{AR} \times \mathrm{SF}$ \\
\hline Cryptosporidium sp. & 27 & $2,065(9-2,740)$ & $167(4-1,659)$ & $284(0-4,505)$ & $<0.01 *$ & $>0.05$ & $<0.05^{*}$ \\
\hline Isospora belli & 15 & $57(11-156)$ & $41(7-187)$ & $31(0-284)$ & $>0.05$ & $>0.05$ & $>0.05$ \\
\hline
\end{tabular}

* Significant difference in the numbers of Cryptosporidium oocysts detected by the Ziehl-Neelsen (ZN) and auramin (AR) compared to the safranin staining method (Friedman nonparametric test).

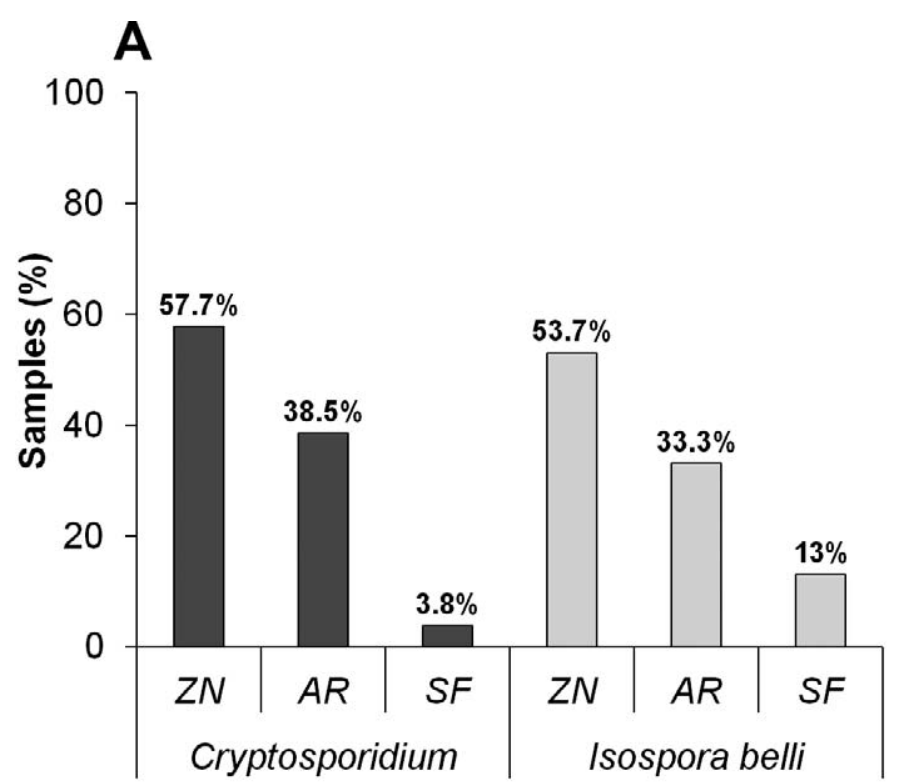

B

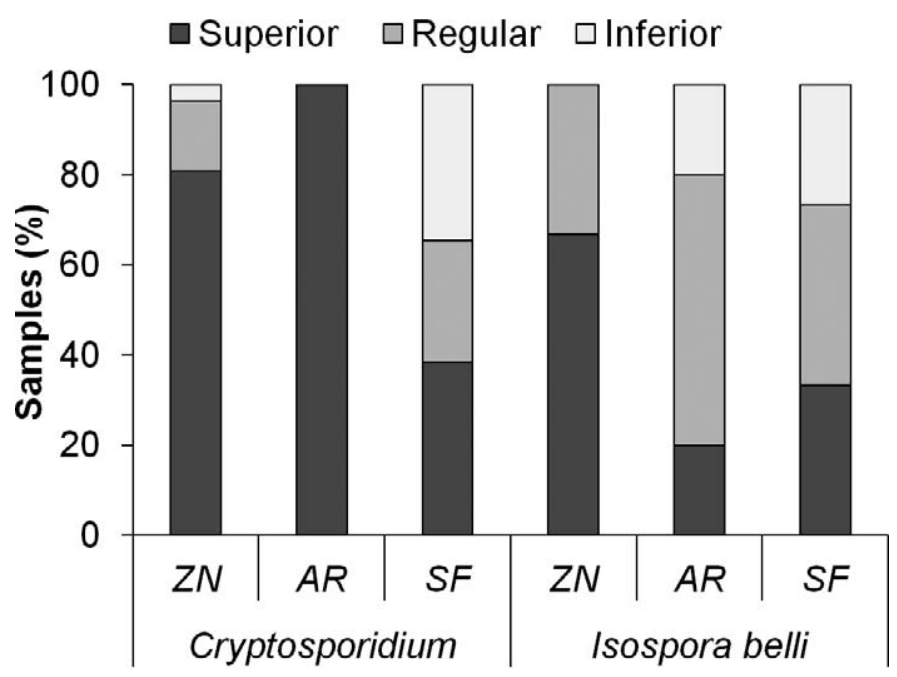

Staining methods

Figure 2. (A) Quantitative evaluation of fecal staining techniques. Twenty-seven Cryptosporidium and 15 Isospora belli fecal samples were concentrated by SC and stained by ZN, AR, or SF. Columns represent the percentual of samples showing higher number of oocysts, analyzed by examining 20 microscopic fields. (B) Qualitative classification of fecal smears. Staining methods were classified as superior, regular, or inferior on the sample analyzed. The huge variation of oocyst loss to the fatty plug among fecal samples indicates the need of examining this area during routine diagnosis in order to avoid false-negative results. This may be most important for low-parasite-load individuals, such as asymptomatic patients, or in those with malabsorption syndrome with a high concentration of fats in their feces. The great variation in the number of oocysts trapped in the fatty layer is a consequence of the individual characteristics of fecal samples. The panel of positive samples used in this study had a wide range of oocyst amounts and variations in the fat content, as they came from different patients. The combination of these 2 factors may account for the differences observed. Indeed, after FE concentration, fatty ring thickness varied according to the sample examined. We have repeated, at least once, the procedure for each sample to check the oocyst loss in the lipid-fat layer, with identical results for individual samples.

We have also observed that smears concentrated by the FE technique more frequently had the presence of "ghost oocysts," which are oocysts with very weak coloration or not stained by fuchsin and which can make diagnosis very difficult. These colorless oocysts have been associated with resolving infections (Harrington, 2009). However, it is possible that residues of ethyl acetate, used in the standard procedure, may have affected the composition of the lipid membrane of the oocysts, changing their tintorial properties. Considering these results in assessing the efficacy of staining methods, the fecal smears were subsequently prepared after concentration by SC.

In relation to staining methods, some studies comparing the sensitivity of ZN to other methods, such as epifluorescence and ELISA, showed no significant differences in Cryptosporidium diagnosis (Kehl et al., 1995; Ignatius and Eisenblatter et al., 1997; Weitzel et al., 2006; Brook et al., 2008; Jayalakshmi et al., 2008). Rigo and Franco (2002) evaluated the staining techniques of modified ZN and acid-fast trichrome (AFT). In their study, the AFT method detected only $42 \%$ of the cryptosporidiosis cases identified by $\mathrm{ZN}$, strengthening the greater sensitivity of $\mathrm{ZN}$ compared to another staining method. Nevertheless, the auramine has been reported to replace carbolfuchsin due to the easy examination of smears with low-power resolution, which decreases the time spent on diagnosis (Nichols and Thom, 1984; Hanscheid et al., 2008). Despite the both quantitatively and qualitatively good performance of AR (also observed herein) for analysis of fecal samples, microscopic examination with $\mathrm{ZN}$ staining is seen as a fast and inexpensive method for diagnosis of

according to the microscopic parameters described in Materials and Methods. 


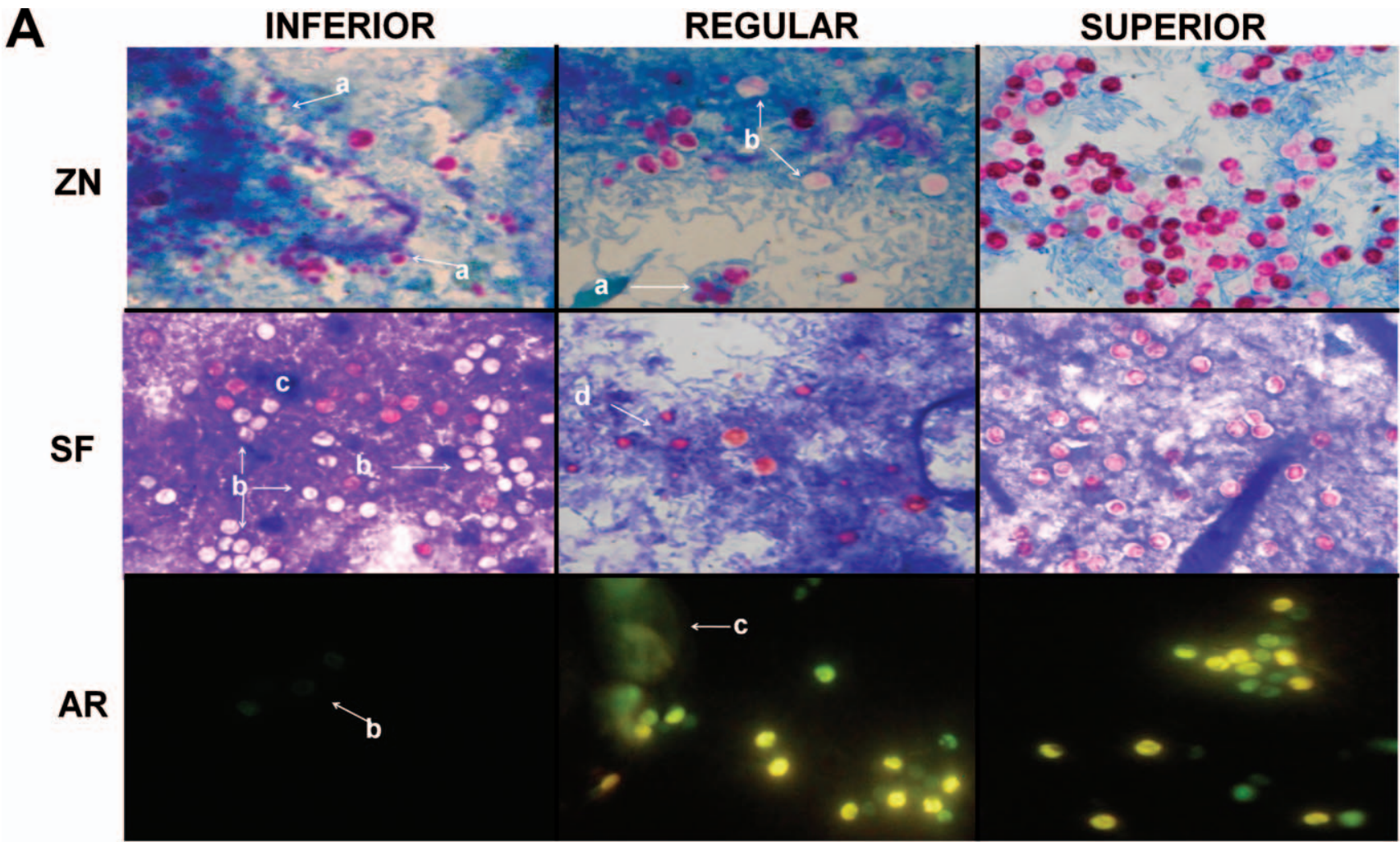

B

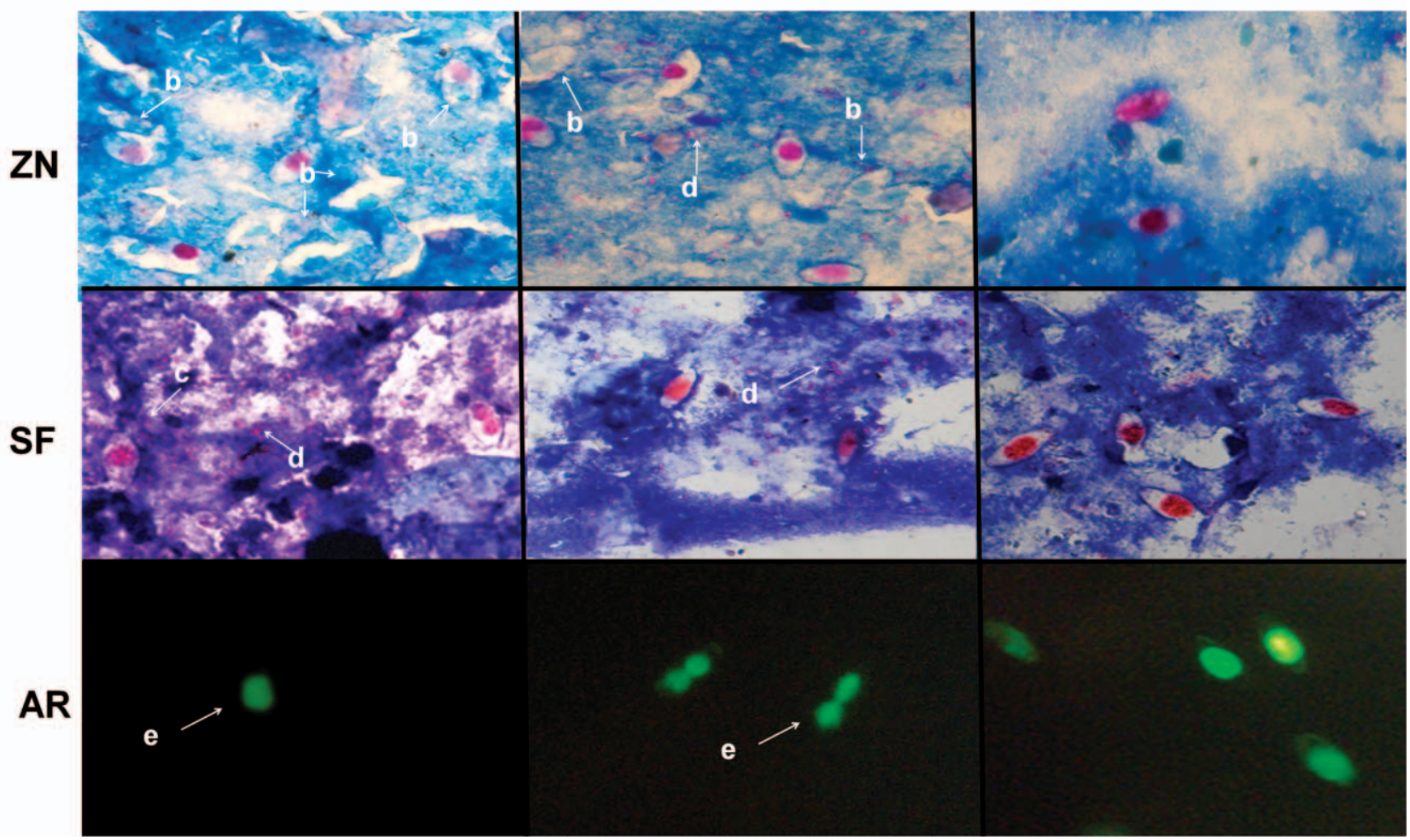

FIgure 3. Photomicrographs of Cryptosporidium (A) and I. belli (B) at $\times 1,000$ and $\times 400$ magnification, respectively, with characteristics used for qualitative assessment of staining methods. INFERIOR fecal smears: presence of yeasts stained with the specific dye (a); low dye uptake by oocysts (b); poor or confusing counterstaining (c); precipitation of dye particles (d); and lack of or reduced oocyst membrane staining (e). REGULAR fecal smears: presence of these undesirable characteristics in less than $50 \%$ of microscopic fields. SUPERIOR fecal smears: easy discrimination of oocysts with less than $25 \%$ of microscopic fields with confounding artifacts. 
both Cryptosporidium and I. belli in immunocompetent and immunocompromised individuals (Ignatius, Lehman et al., 1997; El Naggar et al., 1999; Rigo and Franco, 2002; Magi et al., 2006; Brook et al., 2008). In addition to easy implementation, $\mathrm{ZN}$ staining needs a regular optical microscope for reading the smears while AR staining requires special equipment (a fluorescence microscope) not always available in smaller laboratories, and AR provides slides that cannot be archived.

We have compared 3 different staining methods for coccidians that are commonly used in clinical laboratories. In the study described herein, AR staining displayed the best quality for microscopic detection of Cryptosporidium and was classified as superior for all positive fecal smears examined. However, for $I$. belli detection, AR staining showed the lowest number of smears classified as superior when compared to $\mathrm{ZN}$ and even to SF. This was due to the irregularity of fluorochromo uptake, which increased the difficulty of the microscopical visualization of the parasite, suggesting a lack of uniformity in staining properties within the I. belli oocysts population found in fecal samples and the existence of specific requirements of oocysts staining according to the parasite tested. This may explain the lower number of $I$. belli oocysts detected by AR when compared to $\mathrm{ZN}$ staining and the occurrence of AR-negative slides (zero oocysts detected) in 2 samples.

The variation of coccidian oocysts dying pattern for SF and ZN methods has previously been reported (Pohjola, 1984; Moodley et al., 1991; Elliot et al., 1999; Harrington, 2009). Isospora belli oocysts can also be detected by epifluorescence or lugol wet mounts (Lindsay et al., 1997; Bialek et al., 2002; Pereira et al., 2009) of concentrated fecal samples. However, the former needs an inverted UV light microscope and the latter is less sensitive than tintorial methods due to the thin and fragile I. belli oocyst wall, which can be hidden by other fecal elements and hamper the diagnosis (Lindsay et al., 1997; Pereira et al., 2009).

Many studies evaluating the efficiency of Cryptosporidium diagnostic methods have used the strategy of contaminating fecal samples with purified oocysts or by dividing a single Cryptosporidium positive stool in order to test different techniques (Weber et al., 1991, 1992; Kuczynska and Shelton, 1999; Kvac et al., 2003). These approaches rule out the differences in stool composition or even the nature of coccidian isolates. Different parasites have specific requirements for fecal concentration or oocyst staining, as observed in this study with samples of Cryptosporidium and $I$. belli. A peculiarity of our study was the use of stools from different patients, which reproduces what happens in a routine clinical laboratory. Moreover, the standardization of fecal processing that allows the identification of both parasites may be crucial to the parasitological examination of immunosuppressed individuals.

Laboratory diagnosis of Cryptosporidium in developing countries usually relies on the microscopic examination of stained fecal smears. This study showed a great variability in oocysts power detection by routine parasitological methods, suggesting that SC should be used for fecal concentration rather than FE. Alternatively, because of the high quality of stained fecal smears concentrated by FE, the slides can be prepared using both fecal pellet and fatty plug in order to avoid potential false-negative test results in patients with low parasite excretions. Moreover, analyzing all results together, we propose that a concentration of smears by $\mathrm{SC}$, followed by $\mathrm{ZN}$ staining, can be used as a suitable diagnostic tool in clinical laboratories for both Cryptosporidium and I. belli identification. This will reduce costs as well as the time spent by simultaneously detecting the 2 enteroparasites most frequent in HIV-AIDS patients.

\section{ACKNOWLEDGMENTS}

This work was supported by the Fundação de Amparo à Pesquisa do Estado da Bahia (FAPESB), Conselho Nacional de Desenvolvimento Científico e Tecnológico (CNPq/MCT), and Universidade Federal da Bahia (UFBA), Brazil.

\section{LITERATURE CITED}

Agnamey, P., D. Djeddi, A. Diallo, A. Vanrenterghem, C. Brahimi, C. Costa, And A. Totet. 2010. Childhood cryptosporidiosis: A case report. Journal of Parasitology Research 1: 1-3.

Alemu, A., Y. Shiferaw, G. Getnet, A. Yalew, and Z. Addis. 2011. Opportunistic and other intestinal parasites among HIV/AIDS patients attending Gambi higher clinic in Bahir Dar city, North West Ethiopia. Asian Pacific Journal of Tropical Medicine 4: 661665.

Baxby, D., N. Blundell, And C. A. Hart. 1984. The development and performance of a simple, sensitive method for the detection of Cryptosporidium oocysts in faeces. Journal of Hygiene 93: 317-323.

Bialek, R., N. Binder, K. Dietz, J. Knobloch, and U. E. Zelck. 2002. Comparison of autofluorescence and iodine staining for detection of Isospora belli in feces. American Journal of Tropical Medicine and Hygiene 67: 304-305.

Brook, E. J., R. M. Christley, N. P. French, and C. A. Hart. 2008. Detection of Cryptosporidium oocysts in fresh and frozen cattle faeces: Comparison of three methods. Letters in Applied Microbiology 46: $26-31$.

Cardoso, L. V., K. J. Galisteu, A. S. Junior, L. A. O. Chahla, R. M. S. Canille, M. V. T. Belloto, C. Franco, I. L. Maia, A. R. B. Rossit, AND R. L. D. Machado. 2011. Enteric parasites in HIV-1/AIDS infected patients from a Northwestern São Paulo reference unit in the highly active antiretroviral therapy era. Revista da Sociedade Brasileira de Medicina Tropical 44: 665-669.

Casemore, D. P. 1991. Laboratory methods for diagnosing cryptosporidiosis. Journal of Clinical Pathology 44: 445-451.

, M. Armstrong, And R. L. Sands. 1985. Laboratory diagnosis of cryptosporidiosis. Journal of Clinical Pathology 38: 1337-1341.

Chalmers, R. M., And A. P. Davies. 2010. Minireview: Clinical cryptosporidiosis. Experimental Parasitology 124: 138-146.

Cimerman, S., B. Cimerman, and D. S. Lewi. 1999. Prevalence of intestinal parasitic infections in patients with acquired immunodeficiency syndrome in Brazil. International Journal of Infectious Diseases 3: 203-206.

Clavel, A., A. Arnal, E. Sanchez, M. Varea, J. Quilez, I. Ramirez, And F. J. Castilho. 1996. Comparison of 2 centrifugation procedures in the formalin-ethyl acetate stool concentration technique for the detection of Cryptosporidium oocysts. International Journal for Parasitology 26: 671-672.

Collinet-Adler, S., AND H. D. WArd. 2010. Cryptosporidiosis: Environmental, therapeutic, and preventive challenges. European Journal of Clinical Microbiology and Infectious Diseases 29: $927-$ 935.

El Naggar, H. H., A. E. Handousa, E. M. El Hamshary, and A. M. El Shazly. 1999. Evaluation of five stains in diagnosing human intestinal coccidiosis. Journal of the Egyptian Society of Parasitology 29: $883-891$.

Elgun, G., And I. S. Koltas. 2011. Investigation of Cryptosporidium spp. antigen by ELISA method in stool specimens obtained from patients with diarrhea. Parasitology Research 108: 395-397.

Elliot, A., U. M. Morgan, and R. C. A. Thompson. 1999. Improved staining method for detecting Cryptosporidium oocysts in stool using malachite green. Journal of General and Applied Microbiology 45: 139-142.

Garcia, L. S., And R. Y. Shimizu. 1997. Evaluation of nine immunoassay kits (enzyme immunoassay and direct fluorescence) for detection of 
Giardia lamblia and Cryptosporidium parvum in human fecal specimens. Journal of Clinical Microbiology 35: 1526-1529.

Gupta, S., S. Narang, V. Nunavath, and S. Singh. 2008. Chronic diarrhea in HIV patients: Prevalence of coccidian parasites. Indian Journal of Medical Microbiology 26: 172-175.

Hanscheid, T., J. M. Cristino, and M. J. Salgado. 2008. Screening of auramine-stained smears of all fecal samples is an inexpensive way to increase the detection of coccidial infections. International of Infectious Diseases 12: 47-50.

Harrington, B. J. 2008. Microscopy of 4 pathogenic enteric protozoan parasites: A review. Labmedicine 39: 231-238.

. 2009. The staining of oocysts of Cryptosporidium with the fluorescent brighteners Uvitex 2B and calcofluor white. Labmedicine 40: $219-223$.

Henriksen, S. A., And J. F. L. Pohlenz. 1981. Staining of cryptosporidia by a modified Ziehl-Neelsen technique. Acta Veterinaria Scandinavica 22: 594-596.

Huang, D. B., C. Chappell, and P. C. OKhuysen. 2004. Cryptosporidiosis in children. Seminars in Pediatric Infectious Diseases 15: 253-259.

Huber, F., T. C. Bonfim, and R. S. Gomes. 2003. Comparação da eficiência da técnica de sedimentação pelo formaldeído-éter e da técnica e centrífugo-flutuação modificada na detecção de cistos de Giardia sp e oocistos de Cryptosporidium sp em amostras fecais. Revista Brasileira de Parasitologia Veterinária 12: 135-137.

2004. Comparação da eficiência da coloração pelo método da Safranina a quente e da técnica de centrífugo-flutuação na detecção de oocistos de Cryptosporidium em amostras fecais de animais domésticos. Revista Brasileira de Parasitologia Veterinária 13: 81-84.

Ignatius, R., M. Eisenblatter, T. Regnath, U. Mansmann, U. Futh, H. Hahn, AND J. WaGner. 1997. Efficacy of different methods for detection of low Cryptosporidium parvum oocyst numbers or antigen concentrations in stool specimens. European Journal of Clinical Microbiology and Infectious Diseases 16: 732-736.

- M. Lehman, K. Miksits, T. Regnath, M. Arvand, E. Engelmann, U. Futh, H. Hahn, and J. Wagner. 1997. A new acid fast trichrome stain for simultaneous detection of Cryptosporidium parvum and microsporidial species in stool specimens. Journal of Clinical Microbiology 35: 446-449.

Jayalakshimi, J., B. Appalaraju, and K. Mahadevan. 2008. Evaluation of an enzyme-linked immunoassay for the detection Cryptosporidium antigen in fecal specimens HIV/AIDS patients. Indian Journal of Pathology and Microbiology 51: 137-138.

Kar, S., S. Gawlowska, A. Daugschies, and B. Bangoura. 2011. Quantitative comparison of different purification and detection methods for Cryptosporidium parvum oocysts. Veterinary Parasitology 177: 366-370.

Kehl, K. S. C., H. Cicirello, and P. L. Havens. 1995. Comparison of four different methods for detection of Cryptosporidium species. Journal of Clinical Microbiology 33: 416-418.

Kuczynska, E., and D. R. Shelton. 1999. Method for detection and enumeration of Cryptosporidium parvum oocysts in feces, manures, and soils. Applied and Environmental Microbiology 65: 2820-2826.

Kulkarni, S. V., R. Kairon, S. S. Sane, P. S. Padmawar, V. A. Kale, M. R. Thakar, S. M. Mehendale, and A. R. Risbud. 2009. Opportunistic parasitic infections in HIV/AIDS patients presenting with diarrhea by the level of immunosuppression. Indian Journal of Medical Research 130: 63-66.

Kvac, M., D. Kvetonova, G. Puzova, and O. Ditrich. 2003. Comparison of selected diagnostic methods for identification of Cryptosporidium parvum and Cryptosporidium andersoni in routine examination of faeces. Journal of Veterinary Medicine 50: 405-411.

Lindsay, D. S., J. P. Dubey, and B. L. Blagburn. 1997. Biology of Isospora spp. from humans, nonhuman primates, and domestic animals. Clinical Microbiology Reviews 10: 19-34.
Magi, B., V. Canocchi, G. Tordini, C. Cellesi, and A. Barberi. 2006. Cryptosporidium infection: Diagnostic techniques. Parasitology Research 98: $150-152$

McNabb, S. J., D. M. Hensel, D. F. Welch, H. Heijbel, G. L. Mckee, AND G. R. IstRe. 1985. Comparison of sedimentation and flotation techniques for identification of Cryptosporidium sp. oocysts in a large outbreak of human diarrhea. Journal of Clinical Microbiology 22: $587-589$.

Moodley, D., T. F. Jackson, V. Gathiram, and J. Van Den Ende. 1991. A comparative assessment of commonly employed staining procedures for the diagnosis of cryptosporidiosis. South African Medical Journal 79: $314-317$

Moyo, S. J., N. Gro, M. I. Matee, J. Kitundu, H. Myrmel, H. Mylvaganam, S. Y. Maselle, and N. Langeland. 2011. Age specific aetiological agents of diarrhoea in hospitalized children aged less than five years in Dar es Salaam, Tanzania. BMC Pediatrics 11: 1-6.

Nichols, G., And B. T. Thом. 1984. Screening for Cryptosporidium in stool. Lancet 323: 734-735.

Pereira, D. A., J. Damin, L. M. Lima, and R. W. Uliano. 2009. Isospora belli: Aspectos clínicos e diagnóstico laboratorial. Revista Brasileira de Analises Clínicas 41: 283-286.

Pohjola, S. 1984. Negative staining method with nigrosin for the detection of cryptosporidial oocysts: A comparative study. Research in Veterinary Science 36: 217-219.

Rigo, C. R., And R. M. B. Franco. 2002. Comparação entre os métodos Ziehl-Neelsen modificada e Acid-fast-trichrome para pesquisa fecal de Cryptosporidium parvum e Isospora belli. Revista da Sociedade Brasileira de Medicina Tropical 35: 209-214.

Skotarczak, B. 2010. Progress in the molecular methods for the detection and genetic characterization of Cryptosporidium in water samples. Annals of Agricultural and Environmental Medicine 17: 1-8.

Teixeira, W. F. P., W. M. D. Coelho, C. M. Nunes, and M. V. Meireles. 2011. Detection of Cryptosporidium parvum oocysts in calf fecal samples by direct immunofluorescence assay. Revista Brasileira de Parasitologia Veterinária 20: 269-273.

ten Hove, R. J., L. Van Lieshout, E. A. Brienen, M. A. Perez, and J. J. VERWEIJ. 2008. Real-time polymerase chain reaction for detection of Isospora belli in stool samples. Diagnostic Microbiology and Infectious Disease 61: 280-283.

Truant, A. L., S. H. Elliot, M. T. Kelly, and J. H. Smith. 1981. Comparison of formalin-ethyl ether sedimentation, formalin-ethyl acetate sedimentation, and zinc sulfate flotation techniques for detection of intestinal parasites. Journal of Clinical Microbiology 13: $882-884$.

Vignesh, R., P. Balakrishnan, E. M. Shankar, K. G. Murugavel, S. Hanas, A. J. Cecelia, S. P. Thyagarajan, S. Solomon, and N Kumarasamy. 2007. Short report: High proportion of isosporiasis among HIV-infected patients with diarrhea in Southern India. American Journal of Tropical Medicine and Hygiene 77: 823-824.

Weber, R., R. T. Bryan, H. S. Bishop, S. P. Wahlquist, J. J. Sullivan, AND D. D. JuRANEK. 1991. Threshold of detection of Cryptosporidium oocysts in human stool specimens: Evidence for low sensitivity of current diagnostic methods. Journal of Clinical Microbiology 29: $1323-1327$. - $\longrightarrow$, AND D. D. JURANEK, 1992. Improved stool concentration procedure for detection of Cryptosporidium oocysts in fecal specimens. Journal of Clinical Microbiology 30: 2869-2873.

Weitzel, T., S. Dittrich, I. Mohl, E. Adusu, and T. Jelinek. 2006. Evaluation of seven commercial antigen detection tests for Giardia and Cryptosporidium in stool samples. Clinical Microbiology and Infection 12: 656-659.

Winn, W., S. Allen, W. Janda, E. Koneman, G. Procop, P. SchreckENBERger, AND G. WoOds. 2006. Color atlas and textbook of diagnostic microbiology, 6th ed. Lippincott, Williams and Wilkins, Philadelphia, Pennsylvania, $1565 \mathrm{p}$ 